PROCEEDINGS OF THE

AMERICAN MATHEMATICAL SOCIETY

Volume 127, Number 9, Pages 2685-2695

S 0002-9939(99)05054-6

Article electronically published on April 9, 1999

\title{
TENSOR PRODUCTS OF SUBNORMAL OPERATORS
}

\author{
NATHAN S. FELDMAN
}

(Communicated by David R. Larson)

\begin{abstract}
We shall use a $C^{*}$-algebra approach to study operators of the form $S \otimes N$ where $S$ is subnormal and $N$ is normal. We shall determine the spectral properties for these operators, and find the minimal normal extension and the dual operator. We also give a necessary condition for $C^{*}(S \otimes N)$ to contain a compact operator and a sufficient condition for the algebraic equivalence of $S \otimes N$ and $S \otimes M$.

We also consider the existence of a $*$-homomorphism $\phi: C^{*}(S \otimes T) \rightarrow$ $C^{*}(S)$ satisfying $\phi(S \otimes T)=S$. We shall characterize the operators $T$ such that $\phi$ exists for every operator $S$.

The problem of when $S \otimes N$ is unitarily equivalent to $S \otimes M$ is considered. Complete results are given when $N$ and $M$ are positive operators with finite multiplicity functions and $S$ has compact self-commutator. Some examples are also given.
\end{abstract}

\section{INTRODUCTION}

One of the most well understood classes of subnormal operators is the class of quasinormal operators; this class includes the normal operators and the isometries. Recall that an operator is quasinormal if $S$ and $S^{*} S$ commute. These operators were introduced by Brown [2] in 1953 and have since received considerable attention. The structure theorem that makes these operators prone to study was proven in [2] (also see Conway [7], p.44) and says that a pure operator is quasinormal if and only if it is unitarily equivalent to $U \otimes A$, where $U$ is the unilateral shift and $A$ is a positive one-to-one operator.

Thus, the general feeling is that if a question can be answered for normal operators and for the unilateral shift, then it can also be answered for quasinormal operators. Since quasinormal operators are so well understood, they are often used as a first test to test the resonableness of a conjecture. However, quasinormal operators are very simple in many ways; for instance, their spectrum is always a disk.

In this paper we want to consider a class of subnormal operators that seems to be very apt for study and yet offers much greater diversity than quasinormal operators, namely, operators of the form $S \otimes N$ where $S$ is a subnormal operator

Received by the editors November 20, 1997.

1991 Mathematics Subject Classification. Primary 47B20; Secondary 47A80.

Key words and phrases. Tensor product, subnormal operator, dual operator.

This paper was written while the author was a graduate student at the University of Tennessee working under the direction of Professor John B. Conway. He received partial support from the NSF grant DMS-9401027. 
and $N$ is a normal operator. The idea is that if $S$ is "understood," then the class $S \otimes N$ where $N$ various over the normal operators should offer a rich source of examples and yet still be manageable.

If $S$ and $T$ are subnormal operators, then $S \otimes T$ is also a subnormal operator. For if $N$ and $M$ are normal extensions of $S$ and $T$ respectively, then $N \otimes M$ is a normal extension of $S \otimes T$. In fact, recently J. Stochel [12] proved the converse; that is, if $S \otimes T$ is a subnormal operator, then both $S$ and $T$ must be subnormal. We shall give another proof of a special case of this result.

Now, if $S$ and $T$ are subnormal, what is the minimal normal extension (mne) of $S \otimes T$ ? In particular, if $N=m n e S$ and $M=m n e T$, is $N \otimes M$ the minimal normal extension of $S \otimes T$ ? In general the answer is no (see Conway [6]). In [6] Conway determines the minimal normal extension of $S \otimes S$ when $S$ is the Bergman shift; its description, however, requires approximation theory in several complex variables and the use of disintegration of measures.

Although the class $S \otimes T$ is complicated when $S$ and $T$ are subnormal, we shall see that if one of the factors is normal, then several natural questions are more easily answered. Furthermore, the general tensor product $S \otimes T$ may be obtained by restricting $S \otimes N$ to an invariant subspace, where $N$ is a normal extension of $T$.

We shall show that mne $(S \otimes N)=$ mne $(S) \otimes N$ and $\operatorname{dual}(S \otimes N)=\operatorname{dual}(S) \otimes N^{*}$. Thus one may use this to determine the normal spectrum of $S \otimes N$. However we also obtain the spectral properties by use of the classical Weyl-von Neumann-Berg Theorem and by considering $C^{*}(S \otimes N)$, the $C^{*}$-algebra generated by $S \otimes N$.

An important part of this paper is determining when there exists a $*-$ homomorphism $\phi: C^{*}(S \otimes T) \rightarrow C^{*}(S)$ satisfying $\phi(S \otimes T)=S$. We shall use Voiculescu's Theorem, a non-commutative version of Berg's Theorem, to characterize the operators $T$ with a slightly more general property; namely, there exists a non-zero constant $\alpha$ such that for any operator $S$, there exists a $*$-homomorphism $\phi: C^{*}(S \otimes T) \rightarrow C^{*}(S)$ satisfying $\phi(S \otimes T)=\alpha S$. The operators $T$ for which this holds are precisely those for which there exists a non-zero multiplicative linear functional on $C^{*}(T)$; this includes all hyponormal operators.

This easily and naturally recaptures the result in [12], that subnormality of $S \otimes T$ implies subnormality of $S$, for a large class of operators $T$. The existence of $\phi$ gives a natural way in which to transfer $C^{*}$ properties of the tensor product (such as isometry, normality, subnormality, etc.) to the individual factors.

The problem of when $S \otimes N$ is unitarily equivalent to $S \otimes M$ is of prime importance in understanding these operators. It follows easily from the structure theorem for quasinormal operators that $U \otimes N \cong U \otimes M$ if and only if $|N| \cong|M|$, where $U$ is the unilateral shift and $N, M$ are one-to-one normal operators. We shall prove a version of this for hyponormal operators with compact self-commutator.

If $T$ is a hyponormal operator with compact self-commutator and if $N$ and $M$ are one-to-one normal operators such that either $|N|$ or $|M|$ has a finite multiplicity function, then it is shown that if $T \otimes N \cong T \otimes M$, then $|N| \cong|M|$.

It follows that if $T$ is hyponormal with compact self-commutator, $A$ and $B$ are one-to-one positive operators, one of which has a finite multiplicity function, then $T \otimes A \cong T \otimes B$ if and only if $A \cong B$.

In fact, there are really three different classes of operators here: $S \otimes A$ where $A$ is positive; $S \otimes B$ where $B$ is self-adjoint; and $S \otimes N$ where $N$ is normal. Furthermore, these classes get progressively more complex and, in general, are not equal. If $S$ is the unilateral shift, however, then the three classes all coincide. 


\section{The Minimal NORMAL EXTENSION}

In this section the main result is establishing that mne $(S \otimes N)=m n e(S) \otimes N$. Recall that the self-commutator of an operator $S$ is $\left[S^{*}, S\right]=S^{*} S-S S^{*}$. First we establish when $S \otimes N$ is pure.

Proposition 2.1. If $T$ is any operator and $N$ is normal, then the self-commutator of $T \otimes N$ is $\left[T^{*}, T\right] \otimes N^{*} N$.

Recall that a normal operator is one-to-one if and only if it has dense range.

Proposition 2.2. If $S$ is a subnormal operator and $N$ is a normal operator, then $S \otimes N$ is pure if and only if $S$ is pure and $N$ is one-to-one.

Proof. If $S$ acts on $\mathcal{H}$ and $N$ acts on $\mathcal{K}$, then $T=S \otimes N$ acts on $\mathcal{H} \otimes \mathcal{K}$.

Suppose $S$ is pure and $N$ is one-to-one. To show that $T$ is pure it suffices to show that the smallest invariant subspace of $T$ that contains the range of $\left[T^{*}, T\right]$ is $\mathcal{H} \otimes \mathcal{K}$

If $\mathcal{M}$ is an invariant subspace of $T$ that contains the range of $\left[T^{*}, T\right]=\left[S^{*}, S\right] \otimes$ $N^{*} N$, then $\mathcal{M}$ contains all vectors of the form $\left[T^{*}, T\right](h \otimes k)=\left[S^{*}, S\right] h \otimes N^{*} N k$. Since $N$ has dense range, so does $N^{*} N$. Hence as $k$ varies, we see that $\mathcal{M}$ contains all vectors of the form $\left[S^{*}, S\right] h \otimes k$ for any $h$ and $k$.

Now let $\mathcal{L}=\{x \in \mathcal{H}:$ for every $k \in \mathcal{K}, x \otimes k \in \mathcal{M}\}$. As noted above, the range of $\left[S^{*}, S\right]$ is contained in $\mathcal{L}$. Furthermore, as $\mathcal{M}$ is invariant for $T$, one easily checks that $\mathcal{L}$ is closed and invariant for $S$. Since $S$ is pure we have that $\mathcal{L}=\mathcal{H}$.

Thus, $\mathcal{M}$ contains every vector of the form $h \otimes k$ for any $h \in \mathcal{H}$ and $k \in \mathcal{K}$. Since these vectors have dense linear span in $\mathcal{H} \otimes \mathcal{K}$, we have $\mathcal{M}=\mathcal{H} \otimes \mathcal{K}$. Thus $T$ is pure.

Conversely, if $N$ is not one-to-one, then $S \otimes N$ has a non-trivial kernel; hence it cannot be pure. Or, if $S=S_{1} \oplus M$ where $M$ is normal, then $S \otimes N=\left(S_{1} \otimes N\right) \oplus$ $(M \otimes N)$. Thus, since $M \otimes N$ is normal, $S \otimes N$ is not pure.

Corollary 2.3. If $S$ is a pure subnormal operator and $T$ is any subnormal operator such that mneT is one-to-one, then $S \otimes T$ is a pure subnormal operator.

Proof. Clearly $S \otimes T$ is subnormal. Let $N=m n e T$. Since $S \otimes T$ is the restriction of the pure subnormal operator $S \otimes N$ to an invariant subspace, $S \otimes T$ is also pure.

Proposition 2.4. If $S$ is a subnormal operator and $N$ is a one-to-one normal operator, then mne $(S \otimes N)=\operatorname{mne}(S) \otimes N$.

Proof. If $M=$ mne $S$, then $M \otimes N$ is a normal extension of $S \otimes N$. Suppose $S$ acts on $\mathcal{H}$ and $N$ acts on $\mathcal{K}$. So $S \otimes N$ acts on $\mathcal{H} \otimes \mathcal{K}$. Also, if $M$ acts on $\mathcal{K}_{0}$, then $M \otimes N$ acts on $\mathcal{K}_{0} \otimes \mathcal{K}$. If $\mathcal{M}$ is a reducing subspace of $M \otimes N$ that contains $\mathcal{H} \otimes \mathcal{K}$, then we must show that $\mathcal{M}=\mathcal{K}_{0} \otimes \mathcal{K}$.

Let $\mathcal{L}=\left\{x \in \mathcal{K}_{0}: x \otimes k \in \mathcal{M}\right.$ for every $\left.k \in \mathcal{K}\right\}$. Clearly, $\mathcal{H} \subseteq \mathcal{L}$ and $\mathcal{L}$ is a closed linear subspace of $\mathcal{K}_{0}$. In fact, since $N$ has dense range and $\mathcal{M}$ is a reducing subspace for $M \otimes N$, it follows that $\mathcal{L}$ is a reducing subspace for $M$. Since $\mathcal{H} \subseteq \mathcal{L}$ and $M=$ mneS, it follows that $\mathcal{L}=\mathcal{K}_{0}$. Thus, $h \otimes k \in \mathcal{M}$ for every $h \in \mathcal{K}_{0}$ and $k \in \mathcal{K}$. So, $\mathcal{M}=\mathcal{K}_{0} \otimes \mathcal{K}$ and thus $M \otimes N=\operatorname{mne}(S \otimes N)$. 
Corollary 2.5. If $S$ is a pure subnormal operator and $N$ is a one-to-one normal operator, then dual $(S \otimes N)=\operatorname{dual}(S) \otimes N^{*}$.

Proof. Keeping the notation from Proposition 2.4, we have that $M \otimes N=$ mne $(S \otimes N)$. So, $\operatorname{dual}(S \otimes N)$ is the restriction of $M^{*} \otimes N^{*}$ to $(\mathcal{H} \otimes \mathcal{K})^{\perp}$. Since $(\mathcal{H} \otimes \mathcal{K})^{\perp}=\mathcal{H}^{\perp} \otimes \mathcal{K}$, we see that $\left(M^{*} \otimes N^{*}\right)\left|\left(\mathcal{H}^{\perp} \otimes \mathcal{K}\right)=M^{*}\right| \mathcal{H}^{\perp} \otimes N^{*}=$ $\operatorname{dual}(S) \otimes N^{*}$.

It follows that if $S$ is self-dual and $A$ is self-adjoint, then $S \otimes A$ is also self-dual. Let $m: \mathbb{C}^{2} \rightarrow \mathbb{C}$ be the multiplication map given by $m(z, w)=z w$.

Corollary 2.6. If $S$ is a subnormal operator and $N$ is a one-to-one normal operator, then $\operatorname{svsm}(S \otimes N)=(\mu \times \nu) \circ m^{-1}$ where $\mu=\operatorname{svsmS}$ and $\nu=\operatorname{svsmN}$.

Proof. It follows from Proposition 2.4 that if $M=m n e S$, then

$$
M \otimes N=m n e(S \otimes N) .
$$

Now, if $\mu=\operatorname{svsm} M$ and $\nu=\operatorname{svsm} N$, then it is well known that $\operatorname{svsm}(M \otimes N)=$ $(\mu \times \nu) \circ m^{-1}$.

\section{Spectral Properties}

We now show how to use $C^{*}$-algebra techniques and the classical Weyl-von Neuman-Berg Theorem to establish several properties of $S \otimes N$. We compute the spectral properties, consider when $C^{*}(S \otimes N)$ contains a compact operator, and show the existence of a $*-$ homomorphism from $C^{*}(S \otimes T) \rightarrow C^{*}(S)$ mapping $S \otimes T \mapsto S$ for a large class of operators $T$.

If $N$ is a diagonalizable operator with eigenvalues $\left\{\lambda_{n}\right\}$, then $S \otimes N=\bigoplus_{n} \lambda_{n} S$. Thus $S \otimes N$ is simply a direct sum of multiples of $S$. Clearly, the spectral properties of this direct sum are easily established. For the general case, we shall use the Weyl-von Neumann-Berg Theorem to approximate an arbitrary normal operator by diagonalizable ones.

Two operators $A, B$ are said to be approximately equivalent, denoted $A \sim_{a} B$, if there exist unitaries $U_{n}$ such that $U_{n}^{*} A U_{n} \rightarrow B$ in the norm. We shall need the following two results. The first one is a refined version of the Weyl-von Neumann Theorem due to Berg [1]; also see Davidson [8], p.59.

Theorem 3.1. If $N$ and $M$ are normal operators, then following are equivalent:

(a) $N \sim_{a} M$;

(b) $\sigma_{e}(N)=\sigma_{e}(M)$ and $N, M$ have the same isolated eigenvalues with the same multiplicities.

We also need the following basic result. If $S$ and $T$ are operators, then denote by $C^{*}(S) \approx C^{*}(T)$ the fact that there exists a ${ }^{*}$-isomorphism $\phi: C^{*}(S) \rightarrow C^{*}(T)$ satisfying $\phi(S)=T$.

Proposition 3.2. If $S$ and $T$ are approximately equivalent, then $C^{*}(S) \approx C^{*}(T)$.

Proof. If $U_{n}$ are unitaries such that $U_{n}^{*} S U_{n} \rightarrow T$ in norm, then one easily checks that for each $A \in C^{*}(S)$, the limit of $\left\{U_{n}^{*} A U_{n}\right\}$ exists in norm. If $\phi(A)$ denotes this limit, then $\phi$ is a ${ }^{*}$-homomorphism from $C^{*}(S) \rightarrow C^{*}(T)$ that sends $S$ to $T$. Since one can construct an inverse to $\phi$ in a similar manner, $\phi$ is actually a *-isomorphism. 
We shall also use the following result which we state for convenience.

Theorem 3.3. If $S$ and $T$ are subnormal operators and $C^{*}(S) \approx C^{*}(T)$, then $\sigma_{a p}(S)=\sigma_{a p}(T)$ and $\sigma_{n}(S)=\sigma_{n}(T)$.

The fact that the approximate point spectrum is preserved under $C^{*}$-equivalence actually holds for hyponormal operators and is due to Bunce [4] (also see Conway [7], p. 88). That the normal spectrum is preserved was first shown by Bunce and Deddens [5] in 1977, and independently, and in greater generality, by the author in [9] (also see [10]).

If $K$ and $L$ are two compact subsets of $\mathbb{C}$, then $K L$ denotes the set of all possible products from $K$ and $L$, that is, the range of the multiplication map $m: K \times L \rightarrow \mathbb{C}$ given by $m(z, w)=z w$. Since $K \times L$ is compact, we see that $K L$ is also compact.

Part (a) of the next Theorem is well known and holds for any two operators; see Brown and Pearcy [3].

Theorem 3.4. If $S$ is a subnormal operator and $N$ is a one-to-one normal operator, then the following hold:

(a) $\sigma(S \otimes N)=\sigma(S) \sigma(N)$;

(b) $\sigma_{a p}(S \otimes N)=\sigma_{a p}(S) \sigma(N)$;

(c) $\sigma_{n}(S \otimes N)=\sigma_{n}(S) \sigma(N)$.

Proof. By Theorem 3.1, there exists a diagonal operator $D$ such that $D \sim_{a} N$. Thus, it follows that $S \otimes D \sim_{a} S \otimes N$. In fact if $U_{n}$ are unitaries such that $U_{n}^{*} D U_{n} \rightarrow N$, then $V_{n}^{*}(S \otimes D) V_{n} \rightarrow S \otimes N$ where $V_{n}=I \otimes U_{n}$. Hence Proposition 3.2 implies that $C^{*}(S \otimes D) \approx C^{*}(S \otimes N)$. Thus, Theorem 3.3 gives that the spectrum, approximate point spectrum and normal spectrum of $S \otimes D$ and $S \otimes N$ are the same.

But $D$ is a diagonal operator. If its eigenvalues are $\left\{\lambda_{n}\right\}$, then $S \otimes D=$ $\bigoplus_{n} \lambda_{n} S$. So, for instance, $\left.\sigma_{a p}\left(\bigoplus_{n} \lambda_{n} S\right)=\operatorname{cl}\left[\bigcup_{n} \lambda_{n} \sigma_{a p}(S)\right]=\operatorname{cl}\left[\left\{\lambda_{n}\right\} \sigma_{a p}(S)\right\}\right]=$ $\sigma(D) \sigma_{a p}(S)=\sigma(N) \sigma_{a p}(S)$. So, (b) follows. The others follow similarly.

As mentioned above, part (a) actually holds for any two operators. Also, since Theorem 3.3 holds for the approximate point spectra for hyponormal operators, part (b) also holds whenever $S$ is hyponormal.

We now give another application of Theorem 3.1 to the study of these operators $S \otimes N$. Namely, when does $C^{*}(S \otimes N)$ contain a compact operator? This is not difficult to answer for quasinormal operators. That is, if $U$ is the unilateral shift and $A$ is a one-to-one positive operator, then $C^{*}(U \otimes A)$ contains a non-zero compact operator if and only if $\sigma(A) \neq \sigma_{e}(A)$. In general one direction still holds.

Theorem 3.5. If $S$ is a subnormal operator and $N$ is a normal operator such that $\sigma(N)=\sigma_{e}(N)$, then $C^{*}(S \otimes N)$ does not contain a non-zero compact operator.

Proof. Since $\sigma(N)=\sigma_{e}(N), N$ has no isolated eigenvalues of finite multiplicity. Thus by Theorem 3.1, $N \sim_{a} N^{(2)}=N \oplus N$. It follows that $S \otimes N \sim_{a}(S \otimes N)^{(2)}$. If $\left\{U_{n}\right\}$ are unitaries such that $U_{n}^{*}(S \otimes N) U_{n} \rightarrow(S \otimes N)^{(2)}$, then by Proposition 3.2 the map $\phi: C^{*}(S \otimes N) \rightarrow C^{*}\left((S \otimes N)^{(2)}\right)$ given by $\phi(A)=\lim _{n} U_{n}^{*} A U_{n}$ is a $*$-isomorphism. However, it is also easy to see that $\psi: C^{*}(S \otimes N) \rightarrow C^{*}\left((S \otimes N)^{(2)}\right)$ given by $\psi(A)=A^{(2)}$ is also a $*$-isomorphism. 
Since a $*$-homomorphism is uniquely determined by its action on a generator, we have that $\phi=\psi$. That is, for each $A \in C^{*}(S \otimes N)$ we have $A \sim_{a} \phi(A)=A^{(2)}$. Now if $C^{*}(S \otimes N)$ contains a non-zero compact operator $K$, then it also contains $|K|$. Thus it would follow that $|K| \sim_{a}|K|^{(2)}$. However this contradicts Theorem 3.1 because $|K|$ and $|K|^{(2)}$ have the same isolated eigenvalues although with different multiplicities. Thus there are no non-zero compact operators in $C^{*}(S \otimes N)$.

We now give an example to show that the converse of Theorem 3.5 is not always true.

Example 3.6. There exists a pure subnormal operator $S$ with compact selfcommutator and a positive operator $A$ such that $C^{*}(S \otimes A)$ contains no non-zero compact operators and yet $\sigma(A) \neq \sigma_{e}(A)$.

Proof. Let $B$ be the diagonal operator with eigenvalues $\left\{1,1 / 2, \ldots,(1 / 2)^{n}, \ldots\right\}$. Set $S=U \otimes B$ where $U$ is the unilateral shift. Let $A=\left[M_{x}\right.$ on $\left.L^{2}(1 / 2,1)\right] \oplus C$ where $C$ is any positive one-to-one compact operator with norm one. Since $B \otimes$ $A \cong \bigoplus_{n} 2^{-n} A$, it is easy to check that $\sigma(B \otimes A)=[0,1]=\sigma_{e}(B \otimes A)$. Thus $C^{*}(S \otimes A)=C^{*}(U \otimes(B \otimes A))$ contains no compact operators (using the fact mentioned above about quasinormal operators). But clearly, $\sigma(A) \neq \sigma_{e}(A)$.

We see that $C^{*}$-algebra methods can be useful in studying tensor products. Thus the next question is a natural one.

Question 3.7. If $S$ is a pure subnormal operator and $N, M$ are normal operators, when is $C^{*}(S \otimes N) \approx C^{*}(S \otimes M)$ ?

If $U$ is the unilateral shift, then $C^{*}(U \otimes A) \approx C^{*}(U \otimes B)$ if and only if $\sigma(A)=$ $\sigma(B)$ for one-to-one positive operators $A, B$. Also, it follows from Theorem 3.1 and Proposition 3.2 that if $\sigma(N)=\sigma(M)$, then $C^{*}(S \otimes N) \approx C^{*}(S \otimes M)$. However in general we shall see that the converse is not true; see Example 5.7.

\section{Homomorphisms from $C^{*}(S \otimes T) \rightarrow C^{*}(S)$}

We now consider the existence of a $*$-homomorphism $\phi: C^{*}(S \otimes T) \rightarrow C^{*}(S)$ such that $\phi(S \otimes T)=\alpha S$, for some non-zero $\alpha \in \mathbb{C}$. We shall show that $\phi$ exists whenever $T$ is an operator such that there exists a non-zero multiplicative linear functional on $C^{*}(T)$, and $S$ is arbitrary. This immediately implies that for a large class of operators $T$, if $S \otimes T$ has a certain $C^{*}$-property, such as subnormality, hyponormality, etc., then $S$ also has the same property.

It was shown in [12] that various $C^{*}$-properties (including subnormality) of $S \otimes T$ imply the same property for both $S$ and $T$. The work in [12] is non-trivial and makes use of certain relations between moment problems and operators. Our approach, although it does not apply to every operator $T$, seems more natural and should prove to be useful in other contexts as well.

The following is the main result along these lines.

Theorem 4.1. If $T$ is an operator, then there is a non-zero constant $\alpha \in \mathbb{C}$ such that for any operator $S$, there exists a $*$-homomorphism $\phi: C^{*}(S \otimes T) \rightarrow C^{*}(S)$ such that $\phi(S \otimes T)=\alpha S$ if and only if there exists a non-zero multiplicative linear functional on $C^{*}(T)$.

Remark. Observe that if $\phi$ always exists for any operator $S$, then one direction follows by simply choosing $S$ to be a non-zero operator on a one-dimensional space. 
Lemma 4.2. If $A$ and $B$ are operators and $A^{(\infty)} \sim_{a} A^{(\infty)} \oplus B^{(\infty)}$, then there exists $a *$-homomorphism from $C^{*}(A) \rightarrow C^{*}(B)$ mapping $A \mapsto B$.

Proof. Simply observe that inflation gives that $C^{*}(A) \approx C^{*}\left(A^{(\infty)}\right)$. Also, Proposition 3.2 gives that $C^{*}\left(A^{(\infty)}\right) \approx C^{*}\left(A^{(\infty)} \oplus B^{(\infty)}\right)$. Finally compression shows that there exists a $*$-homomorphism from $C^{*}\left(A^{(\infty)} \oplus B^{(\infty)}\right) \rightarrow C^{*}\left(B^{(\infty)}\right)$. And last, $C^{*}\left(B^{(\infty)}\right) \approx C^{*}(B)$. Composing these maps gives the required $*$-homomorphism.

Proof of Theorem 4.1. First recall Voiculescu's Theorem. It states that if $\rho$ is a non-degenerate representation of a separable $C^{*}$-algebra $\mathcal{A}$ that annihilates the compact operators in $\mathcal{A}$, then $i d \sim_{a} i d \oplus \rho$ where $i d$ denotes the identity representation of $\mathcal{A}$. See [13] or [8], p. 68 .

Let $\psi$ be a non-zero multiplicative linear functional on $C^{*}(T)$. This naturally induces a representation $\rho$ of $C^{*}\left(T^{(\infty)}\right)$ on a separable infinite dimensional Hilbert space; namely $\rho\left(A^{(\infty)}\right)=\psi(A) I$ where $I$ is the identity operator. Since there are no compact operators in $C^{*}\left(T^{(\infty)}\right)$ and $\rho$ is non-degenerate we see that $i d \sim_{a} i d \oplus \rho$. In particular, evaluating at $T$ implies that $T^{(\infty)} \sim_{a} T^{(\infty)} \oplus \alpha I$ where $\alpha=\psi(T)$.

Hence it follows that

$$
S \otimes T^{(\infty)} \sim_{a} S \otimes\left(T^{(\infty)} \oplus \alpha I\right)
$$

So,

$$
(S \otimes T)^{(\infty)} \sim_{a}(S \otimes T)^{(\infty)} \oplus \alpha S^{(\infty)} .
$$

Thus by Lemma 4.2 , there exists a $*$-homomorphism $\phi: C^{*}(S \otimes T) \rightarrow C^{*}(S)$ with $\phi(S \otimes T)=\alpha S$.

It is easy to check that if $\psi$ is a multiplicative linear functional on $C^{*}(T)$, then $\psi(T) \in \sigma_{a p}(T)$. Thus, if $T$ is quasinilpotent, that is $\sigma(T)=(0)$, then $T$ will not satisfy the hypothesis of Theorem 4.1.

However, it is well known that if $T$ is a hyponormal operator and $\alpha \in \sigma_{a p}(T)$, then there exists a multiplicative linear functional $\psi: C^{*}(T) \rightarrow \mathbb{C}$ such that $\psi(T)=$ $\alpha$; see Bunce [4] or Conway [7], p.87.

Corollary 4.3. Let $T$ be a hyponormal operator and $\alpha \in \sigma_{a p}(T)$. If $S$ is any operator, then there exists a $*$-homomorphism $\phi: C^{*}(S \otimes T) \rightarrow C^{*}(S)$ such that $\phi(S \otimes T)=\alpha S$.

One may use Corollary 4.3 to show certain spectral inclusions, such as

$$
\sigma_{a p}(S) \sigma_{a p}(T) \subseteq \sigma_{a p}(S \otimes T)
$$

whenever $S$ and $T$ are hyponormal.

Observe that Corollary 4.3 is easier to prove if $T$ is normal. For suppose that $N$ is normal and $\alpha \in \sigma(N)$. By Theorem 3.1 there exists a diagonal operator $D$ such that $D \sim_{a} N$. But clearly, we may choose $D$ such that $\alpha$ is an eigenvalue for $D$. So, if $S$ is any operator, then $S \otimes N \sim_{a} S \otimes D$. Hence, Proposition 3.2 implies that $C^{*}(S \otimes N) \approx C^{*}(S \otimes D)$. But, since $S \otimes D$ is simply a direct sum of multiples of $S$, compression to a reducing subspace gives a $*$-homomorphism from $C^{*}(S \otimes D) \rightarrow C^{*}(S)$ that maps $S \otimes D \mapsto \alpha S$. Thus by composing we have the required map.

Surely, Corollary 4.3 should prove to be useful in studying tensor products of subnormal and hyponormal operators. 
In [9] (also see [10]) the author showed that subnormality is not only preserved under $*$-homomorphisms but also under certain positive linear maps. Namely, if $S$ is a subnormal operator and $\rho: C^{*}(S) \rightarrow C^{*}(T)$ is a positive (unital) linear map such that $\rho(S)=T$ and $\rho\left(S^{*} S\right)=T^{*} T$, then $T$ is also a subnormal operator. Hence we have the following natural question.

Question 4.4. Can we characterize the operators $T$ such that there exists a nonzero scalar $\alpha \in \mathbb{C}$ with the property that for any operator $S$ there exists a positive (unital) linear map $\rho: C^{*}(S \otimes T) \rightarrow C^{*}(S)$ satisfying $\rho(S \otimes T)=\alpha S$ and $\rho\left(S^{*} S \otimes T^{*} T\right)=|\alpha|^{2} S^{*} S ?$

\section{UNITARY EQUIVALENCE}

In this section we consider the basic question of when $S \otimes N$ is unitarily equivalent to $S \otimes M$. As mentioned in the introduction, if $U$ is the unilateral shift, then $U \otimes N \cong U \otimes M$ if and only if $|N| \cong|M|$. Our approach will be to use the simple fact that if the operators are unitarily equivalent, then so are their selfcommutators.

For any self-adjoint operator $A$, let $n_{A}$ denote the multiplicity function for $A$. Also, any reference to the a.e. behavior of $n_{A}$ naturally means with respect to a scalar valued spectral measure for $A$. We shall say that $A$ has a finite multiplicity function if $n_{A}$ is finite a.e.

The following is our main preliminary result.

Theorem 5.1. Suppose $A, B$ and $C$ are positive operators such that $A$ is compact, $B$ and $C$ are one-to-one and either $B$ or $C$ has a finite multiplicity function. If $A \otimes B \cong A \otimes C$, then $B \cong C$.

An immediate consequence is the following result, which is the main result for this section.

Theorem 5.2. Suppose $S$ is a pure hyponormal operator with compact selfcommutator. If $N$ and $M$ are one-to-one normal operators such that either $|N|$ or $|M|$ has a finite multiplicity function and $S \otimes N \cong S \otimes M$, then $|N| \cong|M|$.

Proof. If $S \otimes N \cong S \otimes M$, then their self-commutators are also unitarily equivalent. So,

$$
\left[S^{*}, S\right] \otimes|N|^{2} \cong\left[S^{*}, S\right] \otimes|M|^{2} .
$$

Thus, since $\left[S^{*}, S\right]$ is a compact positive operator and either $|N|^{2}$ or $|M|^{2}$ has a finite multiplicity function, we must have $|N|^{2} \cong|M|^{2}$. Since this is equivalent to $|N| \cong|M|$, the result follows.

Corollary 5.3. If $S$ is a pure hyponormal operator with compact self-commutator and $A, B$ are positive one-to-one operators one of which has a finite multiplicity function, then $S \otimes A \cong S \otimes B$ if and only if $A \cong B$.

We now proceed toward the proof of Theorem 5.1. We shall need two other results that we state for completeness. First, we shall denote by $\mathcal{A}^{\prime}$ the commutant of an algebra $\mathcal{A}$. Second, recall that a von Neumann algebra $\mathcal{A}$ is finite, if there are no (non-trivial) projections in $\mathcal{A}$ equivalent to the identity, that is, if every isometry in $\mathcal{A}$ is actually a unitary. Clearly, every abelian von Neumann algebra is finite. See [11] for the following result. 
Theorem 5.4 (Kadison \& Singer Theorem). Suppose $A, B$, and $C$ are operators such that $W^{*}(A \oplus B)$ and $W^{*}(A \oplus B)^{\prime}$ are finite von Neumann algebras. If $A \oplus B \cong$ $A \oplus C$, then $B \cong C$.

The next result is a classical result in the theory of von Neumann algebras.

Theorem 5.5. If $A$ is self-adjoint, then $W^{*}(A)^{\prime}$ is a finite von Neumann algebra if and only if the multiplicity function $n_{A}$ is finite a.e.

Proof of Theorem 5.1. Suppose $A \otimes B \cong A \otimes C$ where $A, B$, and $C$ are one-to-one positive operators (the case when $A$ has a kernel follows easily from this case). Suppose that $A$ is compact and $B$ has a finite multiplicity function. We may also suppose that $\|A\|=\|B\|=\|C\|=1$. Let the distinct eigenvalues of $A$ be $\left\{1, \lambda_{1}, \lambda_{2}, \ldots\right\}$, where $1=\lambda_{0}>\lambda_{1}>\lambda_{2}>\ldots$ and where $\lambda_{k}$ has multiplicity $n_{k}$. Thus we have the following:

$$
A \otimes B \cong\left[\begin{array}{cccc}
B^{\left(n_{0}\right)} & 0 & 0 & 0 \\
0 & \lambda_{1} B^{\left(n_{1}\right)} & 0 & 0 \\
0 & 0 & \lambda_{2} B^{\left(n_{2}\right)} & 0 \\
0 & 0 & 0 & \ldots
\end{array}\right] \cong B^{\left(n_{0}\right)} \oplus \bigoplus_{k=1}^{\infty} \lambda_{k} B^{\left(n_{k}\right)}
$$

A similar decomposition holds for $A \otimes C$. As $A \otimes B \cong A \otimes C$, they have equivalent scalar valued spectral measures. Let $\mu=\operatorname{svsm} B$ and $\nu=\operatorname{svsmC}$. Now the only summand of $A \otimes B$ in (1) that puts positive spectral measure on the interval $\left(\lambda_{1}, 1\right]$ is $B^{\left(n_{0}\right)}$. Likewise $C^{\left(n_{0}\right)}$ is the only summand from $A \otimes C$ that contributes to the spectral measure on $\left(\lambda_{1}, 1\right]$. Thus we have that $\mu\left|\left(\lambda_{1}, 1\right] \approx \nu\right|\left(\lambda_{1}, 1\right]$. Also for $t \in\left(\lambda_{1}, 1\right]$, we have $n_{0} n_{B}(t)=n_{A \otimes B}(t)=n_{A \otimes C}(t)=n_{0} n_{C}(t)$. Thus $n_{B}(t)=n_{C}(t)$ for a.e. $t \in\left(\lambda_{1}, 1\right]$.

By considering the spectral projection for $B$ corresponding to the interval $\left(\lambda_{1}, 1\right]$ we can decompose $B$ as $B=B_{1} \oplus B_{2}$ where $\operatorname{svsm} B_{1}=\mu \mid\left(\lambda_{1}, 1\right]$ and $\operatorname{svsm} B_{2}$ is supported on $\left[0, \lambda_{1}\right]$. Also write $C=C_{1} \oplus C_{2}$ in a similar way. Since $B_{1}$ and $C_{1}$ have equivalent spectral measures and the same multiplicity functions, we know that $B_{1} \cong C_{1}$. Thus also, $A \otimes B_{1} \cong A \otimes C_{1}$. So we have the following:

$$
\left(A \otimes B_{1}\right) \oplus\left(A \otimes B_{2}\right) \cong\left(A \otimes C_{1}\right) \oplus\left(A \otimes C_{2}\right) .
$$

Since $B$ has a finite multiplicity function and $A$ is compact, we see that $A \otimes B$ also has a finite multiplicity function. Hence Theorem 5.5 implies that the hypothesis of Theorem 5.4 is satisfied; thus Theorem 5.4 implies that

$$
\left(A \otimes B_{2}\right) \cong\left(A \otimes C_{2}\right)
$$

Now the argument repeats. As $\left(A \otimes B_{2}\right) \cong\left(A \otimes C_{2}\right)$, we have that $\left\|B_{2}\right\|=\left\|C_{2}\right\| \leq$ $\lambda_{1}$. So arguing as above we again see that on the interval $J_{2}=\left(\lambda_{1}\left\|B_{2}\right\|,\left\|B_{2}\right\|\right]=$ $\left(\lambda_{1}\left\|C_{2}\right\|,\left\|C_{2}\right\|\right], B_{2}$ and $C_{2}$ have equivalent spectral measures and the same multiplicity function. Hence we can write $B_{2}=B_{3} \oplus B_{4}$ and $C_{2}=C_{3} \oplus C_{4}$, where $B_{3}$ and $C_{3}$ have (equivalent) spectral measures carried by $J_{2}$ and $B_{4}$ and $C_{4}$ have spectral measures supported on $\left[0, \lambda_{1}\left\|B_{2}\right\|\right]=\left[0, \lambda_{1}\left\|C_{2}\right\|\right]$. Thus, $\left(A \otimes B_{3}\right) \oplus\left(A \otimes B_{4}\right) \cong$ $\left(A \otimes C_{3}\right) \oplus\left(A \otimes C_{4}\right)$ and since $B_{3} \cong C_{3}$ and $B$ has a finite multiplicity function, Theorem 5.4 gives that $A \otimes B_{4} \cong A \otimes C_{4}$. Also, $\left\|B_{4}\right\|=\left\|C_{4}\right\| \leq \lambda_{1}\left\|B_{2}\right\| \leq \lambda_{1}^{2}$. Continuing in this manner and since $\lambda_{1}^{n} \rightarrow 0$, we see that $B$ and $C$ have equivalent spectral measures and the same multiplicity functions on $(0,1]$. Since the operators $B$ and $C$ are one-to-one, it follows that $B \cong C$. 
We now give an example of positive operators $A, B$ and $C$ with $A$ compact showing that the multiplicity assumption in Theorem 5.1 is necessary.

Example 5.6. Let A be the diagonal operator with entries $\left\{1,1 / 2, \ldots,(1 / 2)^{n}, \ldots\right\}$. Also for $0 \leq \delta<1$, let $B_{\delta}$ be $M_{x}$ on $L^{2}(\delta, 1)$. If $0 \leq \delta, \epsilon \leq 1 / 2$, then $A \otimes B_{\delta}^{(\infty)} \cong$ $A \otimes B_{\epsilon}^{(\infty)}$. However, if $\delta \neq \epsilon$, then $B_{\delta}^{(\infty)}$ is not unitarily equivalent to $B_{\epsilon}^{(\infty)}$.

Proof. This follows because for any $0 \leq \delta \leq 1 / 2$, the scalar valued spectral measure of $A \otimes B_{\delta}^{(\infty)}$ is Lebesgue measure on $(0,1)$ and its multiplicity function is identically infinite. This may be verified using a decomposition as in (1) above.

We now use Example 5.6 to construct another example illustrating how far things can go wrong in Corollary 5.3 if $A$ or $B$ does not have finite multiplicity.

Example 5.7. There exists a pure subnormal operator $S$ with compact selfcommutator and two positive one-to-one operators $B, C$ such that $S \otimes B \cong S \otimes C$ and yet $\sigma(B) \neq \sigma(C)$.

Proof. Keep the same notation as in Example 5.6. Set $S=U \otimes A$ where $U$ is the unilateral shift. Thus $S$ is a pure subnormal operator with compact self-commutator. Now, if $\delta \neq \epsilon, 0 \leq \delta, \epsilon \leq 1 / 2$ and we set $B=B_{\delta}^{(\infty)}$ and $C=B_{\epsilon}^{(\infty)}$, then we have (from Example 5.6) that $A \otimes B \cong A \otimes C$. Hence $U \otimes(A \otimes B) \cong U \otimes(A \otimes C)$. Thus, $S \otimes B \cong S \otimes C$ and yet $B$ and $C$ do not even have the same spectrum.

We now give one more example showing that the compactness of the selfcommutator is necessary in Corollary 5.3.

Example 5.8. There exists a pure subnormal operator $S$ and positive one-to-one compact operators $A, B$ such that $S \otimes A \cong S \otimes B$ and yet $\sigma(A) \neq \sigma(B)$.

Proof. Again, keep the same notation as in Example 5.6. Fix $0<\delta \leq 1 / 2$. Let $S=U \otimes B_{\delta}^{(\infty)}$, where $U$ is the unilateral shift. Now, $A$ is a diagonal operator and let $B=A \oplus K$ for any positive one-to-one compact operator $K$ with $\|K\| \leq 1$. Thus, it follows as in Example 5.6 that $S \otimes A \cong S \otimes B$ and yet $\sigma(A) \neq \sigma(B)$.

Question 5.9. Can one give necessary and sufficient conditions for $S \otimes N$ to be unitarily equivalent to $S \otimes M$ where $S$ is subnormal and $N, M$ are normal operators?

\section{REFERENCES}

[1] I.D. Berg, An extension of the Weyl-von Neumann Theorem to normal operators, Trans. Amer. Math. Soc. 160 (1971), 365-371. MR 44:840

[2] A. Brown, On a class of operators, Proc. Amer. Math. Soc. 4 (1953), 723-728. MR 15:538c

[3] A. Brown and C. Pearcy, Spectra of tensor products of operators, Proc. Amer. Math. Soc. 17 (1966), 162-166. MR 32:6218

[4] J.W. Bunce, Characters on singly generated $C^{*}$-algebras, Proc. Amer. Math. Soc. 25 (1970), 297-303. MR 41:4258

[5] J.W. Bunce and J.A. Deddens, On the normal spectrum of a subnormal operator, Proc. Amer. Math. Soc. 63 (1977), 107-110. MR 55:8863

[6] J.B. Conway, Towards a functional calculus for subnormal tuples: the minimal normal extension and approximation in several complex variables, Proc. Symp. Pure Math., vol. 51, part 1, Amer. Math. Soc., Providence, R.I., 1990, p. 105-112. CMP 91:03

[7] J.B. Conway, The Theory of Subnormal Operators, Amer. Math. Soc., Providence, RI, 1991. MR 92h: 47026

[8] K.R. Davidson, $C^{*}$-algebras by Example, Amer. Math. Soc., Providence, RI, 1996. MR 97i: 46095 
[9] N.S. Feldman, The self-commutator of a subnormal operator, Ph.D. Thesis, University of Tennessee, 1997.

[10] N.S. Feldman, Essentially subnormal operators, to appear in Proc. Amer. Math. Soc.

[11] R.V. Kadison and I.M. Singer, Three Test Problems in Operator Theory, Pacific J. Math. 7 (1957), 1101-1106. MR 19:1066e

[12] J. Stochel, Seminormality of operators from their tensor product, Proc. Amer. Math. Soc. 124 (1996), 135-140. MR 96d:47030

[13] D.V. Voiculescu, A non-commutative Weyl-von Neumann Theorem, Rev. Roum. Pures Appl. 21 (1976), 97-113. MR 54:3427

Department of Mathematics, Michigan State University, East Lansing, Michigan 48824-1027

E-mail address: feldman@math.msu.edu 